\title{
Clinical Study \\ X-Linked Retinoschisis in Juveniles: Follow-Up by Optical Coherence Tomography
}

\author{
Qin-rui Hu, Lv-zhen Huang, Xiao-li Chen, Hui-ka Xia, Tian-qi Li, and Xiao-xin Li \\ Department of Ophthalmology, Peking University People's Hospital, Key Laboratory of Vision Loss and Restoration, \\ Ministry of Education, Beijing Key Laboratory for the Diagnosis and Treatment of Retinal and Choroid Diseases, Beijing, China
}

Correspondence should be addressed to Xiao-xin Li; drlixiaoxin@163.com

Received 25 October 2016; Revised 13 December 2016; Accepted 23 January 2017; Published 14 February 2017

Academic Editor: Susmito Biswas

Copyright (C) 2017 Qin-rui Hu et al. This is an open access article distributed under the Creative Commons Attribution License, which permits unrestricted use, distribution, and reproduction in any medium, provided the original work is properly cited.

Purpose. To explore the structural progression of X-linked retinoschisis (XLRS) in patients by using spectral-domain optical coherence tomography (SD-OCT). Design. Retrospective, observational study. Methods. Patients who were diagnosed with XLRS by genetic testing underwent comprehensive ophthalmological examinations from December 2014 to October 2016. Each eye was measured by SD-OCT using the same clinical protocol. A correlation between best-corrected visual acuity (VA) and SD-OCT measurements was observed. Results. Six patients demonstrated retinoschisis (12 eyes) and typical foveal cyst-like cavities (10 eyes) on SD-OCT images with a mean logMAR VA of 0.48 . The median age was 7.5 years at the initial visit. Their foveal retinal thickness $(516.9 \mu \mathrm{m})$ and choroid thickness $(351.4 \mu \mathrm{m})$ decreased at a rate of 38.1 and $7.5 \mu \mathrm{m}$, respectively, at the 10.5-month follow-up visit; however, there were no significant differences $(P=0.622$ and $P=0.406$, resp.). There was no significant correlation between VA, the foveal retinal thickness, and subfoveal choroid thickness. Conclusions. SD-OCT images for XLRS patients during the juvenile period revealed no significant changes in the fundus structure, including the foveal retinal thickness and choroid thickness within one-year follow-up. There was a lack of correlation between VA, foveal retinal thickness, and subfoveal choroid thickness.

\section{Introduction}

$\mathrm{X}$-linked retinoschisis (XLRS) is an inherited vitreoretinal dystrophy and is characterized by foveal schisis in patients [1-3]. The prevalence of XLRS varies from 1:5000 to 1:25000 $[2,4-6]$. OCT is a safe and noninvasive procedure to diagnose and monitor patients with XLRS [7]. Although the macular anatomy has been well studied using OCT, the follow-up and quantification of foveal retinal thickness and choroid thickness in XLRS patients have not been well documented [8].

The goal of this study was to use SD-OCT to evaluate the progression of structural changes in the retina in XLRS patients and their correlation with visual function during the follow-up period.

\section{Methods}

The study procedures were performed in accordance with institutional guidelines and the Declaration of Helsinki.
Informed consent was obtained from all patients after a full explanation of the procedures.

All patients were diagnosed with X-linked retinoschisis by genetic testing. The following data about patients were collected: funduscopic examination data, measurements of visual acuity (VA) with a standard $\log$ MAR visual acuity chart, and SD-OCT examination data. The images were acquired using a Cirrus HD-OCT unit (Cirrus HD-OCT; Carl Zeiss Meditec) with line scan (6 $\mathrm{mm}$ on the retina), a wavelength of $840 \mathrm{~nm}$, and an axial resolution of $5 \mu \mathrm{m}$. Multiple measures were obtained for the foveal retinal and subfoveal choroid thicknesses. Clinical therapies were recorded during the visit.

A paired Student's $t$-test was performed to compare paired clinical data for foveal retinal and subfoveal choroidal thickness measurements for the subjects at the first and last examination (SPSS 16.0). A Pearson test was used to correlate VA with the foveal retinal thickness and the choroid thickness. A $P$ value of 0.05 was considered statistically significant. 


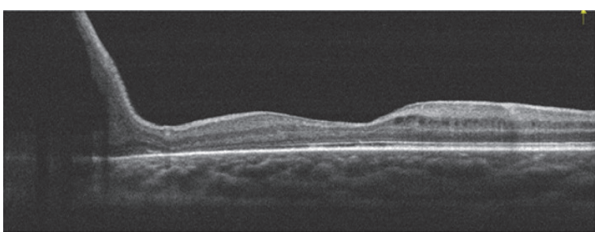

(a)

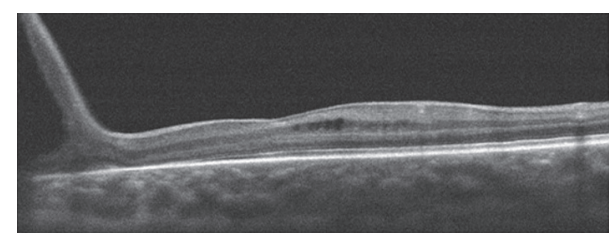

(b)

FIGURE 1: Optical coherence tomographic images for one patient. (a) Vertical images revealed a relatively normal foveal retinal thickness with peripheral retinoschisis of the fundus. (b) Image of the same eye 1.5 years later. The fundus structure did not have obvious changes.

\section{Results}

The clinical characteristics are summarized in Table 1 from December 2014 to October 2016. Eight male patients were enrolled in the study. Two patients were excluded because of vitreous hemorrhage and peripheral retinal detachment by trauma. Therapeutic scleral buckling procedures were performed for these patients. The median age of the 6 patients at the first visit was 7.5 years, with a range from 5 years to 11 years. The average visual acuity was $0.48 \pm 0.21$ (median: 0.45 ) at the first visit, whereas at the last visit it was $0.46 \pm$ 0.28 (median: 0.35 ) after an average of 10.5-month follow-up period. The median foveal retinal thickness at the first visit was $566.5 \mu \mathrm{m}$ (the last visit: $454.5 \mu \mathrm{m}$ ) ranging from $147.0 \mu \mathrm{m}$ to $869.0 \mu \mathrm{m}$. The median subfoveal choroid thicknesses were $343.5 \mu \mathrm{m}$ and $351.5 \mu \mathrm{m}$ at the second visit. Of the 6 patients, there were typical foveal cyst-like cavities in 10 eyes $(83.3 \%)$. Schisis in the INL was observed in all 12 eyes (100\%). GCL schisis was observed frequently $(83.3 \%)$ in 10 eyes, slightly higher than outer plexiform layer (OPL) schisis observed in 8 eyes $(66.7 \%)$.

The average foveal retinal thickness $(516.9 \mu \mathrm{m})$ and subfoveal choroid thickness $(351.4 \mu \mathrm{m})$ decreased at a rate of 38.1 and $7.5 \mu \mathrm{m}$, respectively, during the follow-up period, but there were no significant differences between the two visits (foveal retinal thickness, $P=0.622$; subfoveal choroid thickness, $P=0.406$ ). There was a lack of correlation between VA, foveal retinal thickness, and subfoveal choroid thickness (Table 2).

\section{Discussion}

In our study, VA remained stable during the follow-up period and did not correlate with either foveal retinal thickness or choroid thickness in XLRS patients, except for two patients who lost their vison because of vitreous hemorrhage and retinal detachment. These findings are consistent with previous reports that visual acuity is stable if no secondary event occurs $[2,9]$.

Our study found that both the inner and outer retinal structures of XLRS patients were affected. SD-OCT showed that the INL (100\%, 12 eyes) was the most prevalent area of schisis or defect. The GCL was also frequently affected (83.3\%). These results are supported by previous pathology based studies, which demonstrated that inner retinal abnormalities were the main manifestation of XLRS $[10,11]$.
The average foveal retinal thickness was $516.9 \mu \mathrm{m}$ and decreased to $478.8 \mu \mathrm{m}$ in our study. Foveal retinal thickness remained relatively stable during the follow-up period. The foveal cyst-like lesions underwent visible reduction only in a few cases, but the tiny cystic change was hard to clearly define and quantify, because a completely consistent baseline SD-OCT scan was nearly impossible. A previous study described progressive changes in foveal thickness and that foveal thickness was reduced to below normal over time; therefore, macular cystic-like lesions would no longer be apparent and atrophic-appearing lesions could be observed $[4,12]$. Middle-aged and older patients often presented a nonspecific atrophic appearance of macular lesions $[1,9]$. The present study suggests that the retina remains structurally stable in adolescence and atrophy may appear for decades. Monitoring macular change is feasible once a year using SDOCT.

In the study by Yang et al., the average subfoveal choroidal thicknesses were approximately $358.0 \mu \mathrm{m}$ and $35 \mu \mathrm{m}$ thicker in the patient group than in the normal control group, but it failed to achieve statistical significance $(P=0.084)$ [13]. In our study, the average subfoveal choroidal thickness was $351.4 \mu \mathrm{m}$, which was similar to the study above. Moreover, at the end of nearly one-year follow-up, the subfoveal choroidal thickness was minimally decreased by $7.5 \mu \mathrm{m}(P=0.406)$. Thus, we conclude that the subfoveal choroidal thickness remains constant in adolescent XLRS patients.

However, XLRS is characterized by a high degree of clinical variability between individuals. In our study, one patient (Figure 1) showed a relatively normal SD-OCT appearance in the macular area, whereas the main pathological changes existed in the peripheral fundus. In this case, SD-OCT only provided a subtle diagnostic clue, which was not sufficient to monitor the condition. More evidence was necessary to make the diagnosis, including flash-electroretinogram (ERG) and genetic testing. For peripheral retinoschisis, a wide-field SD-OCT imaging technique or ERG may be good auxiliary methods to monitor progress. According to a previous study, wide-field SD-OCT allowed the simultaneous visualization of the macular and extra macular regions, necessary for understanding complex retinal anatomy with diffuse or multifocal schisis involving multiple retinal layers [8]. Widefield SD-OCT scans may be a promising tool in XLRS clinical trials. For another patient (Figure 2), the SD-OCT showed a typical appearance of foveal cystoid spaces in the binocular. However, his ERG results were normal in both eyes with both 


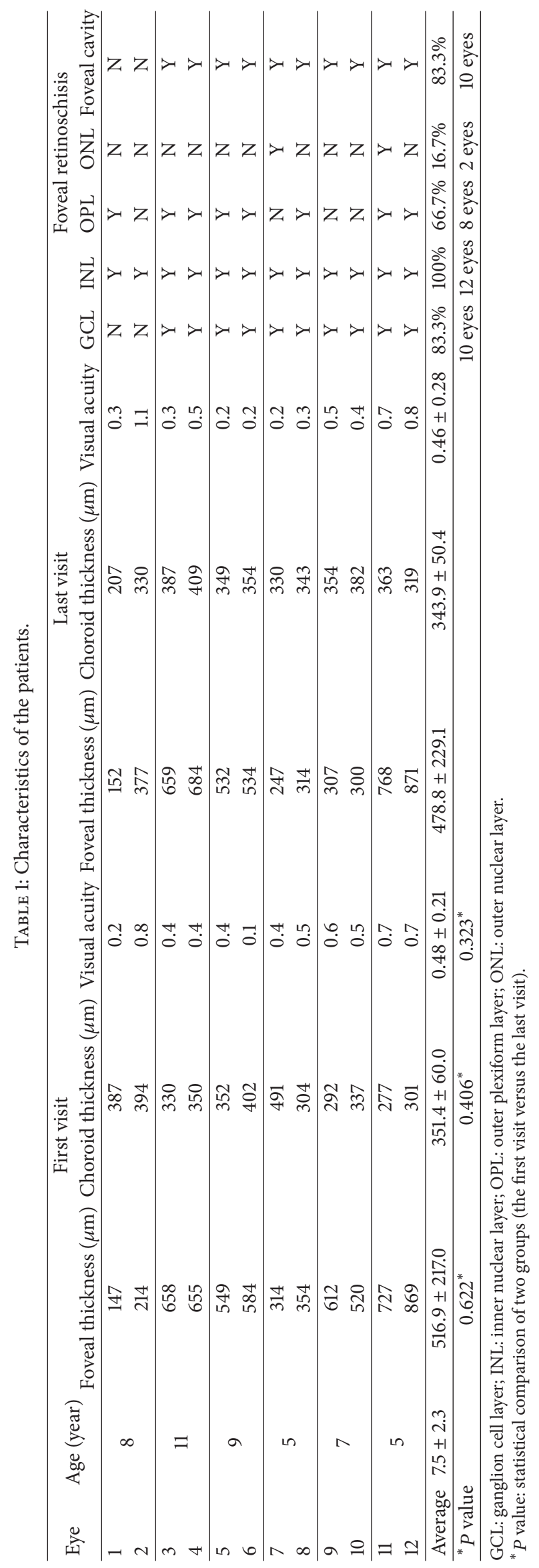


TABLE 2: Correlations of clinical and optical coherence tomographic characteristics of the patients.

\begin{tabular}{|c|c|c|c|c|}
\hline & & Visual acuity & Foveal thickness & Choroid thickness \\
\hline \multirow{3}{*}{ Visual acuity } & Pearson correlation & 1 & 0.280 & -0.190 \\
\hline & Sig. (2-tailed) & & 0.185 & 0.373 \\
\hline & $N$ & 24 & 24 & 24 \\
\hline \multirow{3}{*}{ Foveal thickness } & Pearson correlation & 0.280 & 1 & -0.092 \\
\hline & Sig. (2-tailed) & 0.185 & & 0.670 \\
\hline & $N$ & 24 & 24 & 24 \\
\hline \multirow{3}{*}{ Choroid thickness } & Pearson correlation & -0.190 & -0.092 & 1 \\
\hline & Sig. (2-tailed) & 0.373 & 0.670 & \\
\hline & $N$ & 24 & 24 & 24 \\
\hline
\end{tabular}

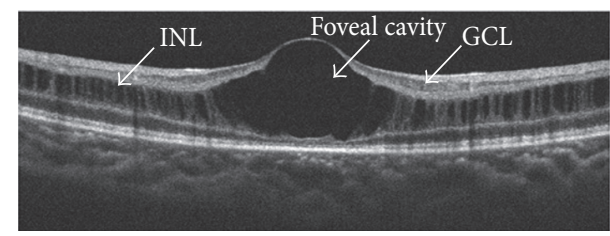

(a)

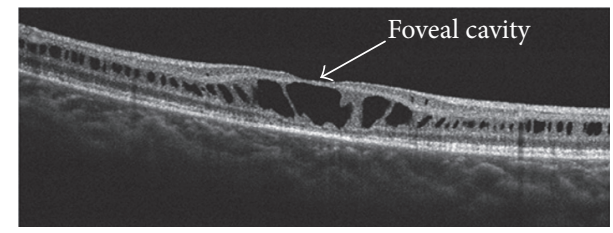

(b)

FIGURE 2: Optical coherence tomographic images for another patient. (a) Vertical images revealed defects in the ganglion cell layer (GCL) and inner nuclear layer (INL). (b) Image of the same eye 1 year later. Concave deformations appeared on the surface of the foveal cystic cavity.

a- and b-wave amplitudes within the normal range. On the SD-OCT of the fovea, although the center foveal convexity dropped over time to a slight concave profile, the peripheral retina change was not significant. In these cases, a single measurement with SD-OCT was not sufficient to evaluate the progress of the fundus structure and eye function; therefore, integrated assessments are recommended.

Choriocapillaris provides the necessary nutrients for the outer retina. However, the actual relationship and order of degeneration in the choroidal and retina interface in certain diseases remain not fully understood $[14,15]$. A previous study found that choriocapillaris breakdown preceded retinal degeneration in age-related macular degeneration [16]. Data indicated that choriocapillaris breakdown occurred during normal aging and preceded degeneration of the retinal pigment epithelium (RPE) and retina. In the present study, we identified a correlation between the structure and function in juveniles. However, no correlation was found between the foveal retinal thickness, the choroidal thickness, and the visual acuity. We propose that this result is related to continuing ocular axial length growth in the sample of children with XLRS. Thirteen is considered the age marking the end of growth of the eye axial length $[2,17,18]$. In an older patient group ( $>13$ years) in a study of XLRS by Vincent A, the refractive error was significantly more hypermetropic and the axial length was significantly shorter than the normal adult group [18]. The overall clinical picture may resemble atrophic macular degeneration in older individuals [19]. In our study, the patients were at a stage in which the general structure of the fovea and subfoveal choroid would not have dramatic atrophic changes. Slight changes of the subfoveal choroid thickness show no significant correlation with damage to vison function and obvious transformation may not be present for decades. This result was also consistent with previous studies $[9,13]$. Although some subtle changes could be found, the short-term follow-up of less than one year would be unlikely to detect obvious changes in the natural course of XLRS. Particular attention should be given to the investigation of different stages for XLRS patients who may reveal a possible correlation between the retina, choroidal, and eye function.

Two patients lost their vision because of vitreous hemorrhage caused by trauma. A similar outcome was also noted by a previous study [2]. As vitreous hemorrhage is mainly caused by the rupture of unsupported blood vessels or preretinal neovascularization $[19,20]$, avoiding intense activity should be advised for XLRS patients.

There are several limitations of this study. It is possible that the present results do not reflect the exact changes in measurements, because a very small number of cases were recruited in this study. The patients in the present study were followed up for a short time within one year, whereas the condition remained clinically stable in the younger age group. Moreover, it is difficult to obtain consistent measurements on the SD-OCT scan at different time points when there is significant distortion of the normal anatomy. Therefore, measurements with the SD-OCT may be prone to bias.

In conclusion, SD-OCT may be used to monitor structural changes over time in patients with XLRS, but further evidence is required to ascertain any biomarkers of disease progression. In the present study, we found that schisis occurred most frequently at the INL and GCL. No obvious structural changes were observed during the follow-up period. XLRS complications are variable in the clinic, and trauma should be avoided, particularly in the adolescent. Timely and targeted measures should be considered when 
managing complications to prevent progressive visual deterioration and improve the visual prognosis.

\section{Disclosure}

Funding institutions had no role in the study design, data collection and analysis, the decision to publish, or preparation of the manuscript.

\section{Competing Interests}

The authors declare no competing financial interests.

\section{Authors' Contributions}

Qin-rui $\mathrm{Hu}$ and Lv-zhen Huang contributed equally to this paper and are cofirst authors.

\section{Acknowledgments}

This work was supported by the National Basic Research Program of China (973 Program, 2011CB510200) and the National Natural Science Foundation of China, Grant no. 81470649 .

\section{References}

[1] U. Kellner, S. Brümmer, M. H. Foerster, and A. Wessing, "Xlinked congenital retinoschisis," Graefe's Archive for Clinical and Experimental Ophthalmology, vol. 228, no. 5, pp. 432-437, 1990.

[2] N. D. L. George, J. R. W. Yates, K. Bradshaw, and A. T. Moore, "Infantile presentation of X linked retinoschisis," British Journal of Ophthalmology, vol. 79, no. 7, pp. 653-657, 1995.

[3] H. Forsius, U. Krause, J. Helve et al., "Visual acuity in 183 cases of X-chromosomal retinoschisis," Canadian Journal of Ophthalmology, vol. 8, no. 3, pp. 385-393, 1973.

[4] The Retinoschisis Consortium, "Functional implications of the spectrum of mutations found in 234 cases with X-linked juvenile retinoschisis," Human Molecular Genetics, vol. 7, no. 7, pp. 1185-1192, 1998.

[5] T. Alitalo, H. Forsius, J. Kärnä et al., "Linkage relationships and gene order around the locus for X-linked retinoschisis," American Journal of Human Genetics, vol. 43, no. 4, pp. 476483, 1988.

[6] R. S. Molday, U. Kellner, and B. H. F. Weber, "X-linked juvenile retinoschisis: clinical diagnosis, genetic analysis, and molecular mechanisms," Progress in Retinal and Eye Research, vol. 31, no. 3, pp. 195-212, 2012.

[7] M. A. Apushkin, G. A. Fishman, and M. J. Janowicz, "Correlation of optical coherence tomography findings with visual acuity and macular lesions in patients with X-linked retinoschisis," Ophthalmology, vol. 112, no. 3, pp. 495-501, 2005.

[8] N. Z. Gregori, B. L. Lam, G. Gregori et al., "Wide-field spectraldomain optical coherence tomography in patients and carriers of X-linked retinoschisis," Ophthalmology, vol. 120, no. 1, pp. 169-174, 2013.

[9] S. Kjellström, C. Vijayasarathy, V. Ponjavic, P. A. Sieving, and S. Andréasson, "Long-term 12 year follow-up of X-linked congenital retinoschisis," Ophthalmic Genetics, vol. 31, no. 3, pp. 114-125, 2010.
[10] W. A. Manschot, "Pathology of hereditary juvenile retinoschisis," Archives of Ophthalmology, vol. 88, no. 2, pp. 131-138, 1972.

[11] M. Yanoff, E. K. Rahn, and L. E. Zimmerman, "Histopathology of Juvenile Retinoschisis," Archives of Ophthalmology, vol. 79, no. 1, pp. 49-53, 1968.

[12] Y. Takada, R. N. Fariss, A. Tanikawa et al., "A retinal neuronal developmental wave of retinoschisin expression begins in ganglion cells during layer formation," Investigative Ophthalmology and Visual Science, vol. 45, no. 9, pp. 3302-3312, 2004.

[13] H. S. Yang, J. B. Lee, Y. H. Yoon, and J. Y. Lee, "Correlation between spectral-domain OCT findings and visual acuity in $\mathrm{X}$-linked retinoschisis," Investigative Ophthalmology and Visual Science, vol. 55, no. 5, pp. 3029-3036, 2014.

[14] P. Amalric, "[Changes in the choriocapillaris and the pigment epithelium during development of retinoschisis]," Bulletin de la Societe Belge d'Ophtalmologie, vol. 198, no. 2, pp. 7-14, 1981.

[15] G. Lutty, J. Grunwald, A. B. Majji, M. Uyama, and S. Yoneya, "Changes in choriocapillaris and retinal pigment epithelium in age-related macular degeneration," Molecular Vision, vol. 5, article 35, 1999.

[16] A. Biesemeier, T. Taubitz, S. Julien, E. Yoeruek, and U. Schraermeyer, "Choriocapillaris breakdown precedes retinal degeneration in age-related macular degeneration," Neurobiology of Aging, vol. 35, no. 11, pp. 2562-2573, 2014.

[17] R. Mendoza-Londono, K. T. Hiriyanna, E. L. Bingham et al., "A Colombian family with X-linked juvenile retinoschisis with three affected females: finding of a frameshift mutation," Ophthalmic Genetics, vol. 20, no. 1, pp. 37-43, 1999.

[18] A. Vincent, A. G. Robson, M. M. Neveu et al., "A phenotypegenotype correlation study of X-linked retinoschisis," Ophthalmology, vol. 120, no. 7, pp. 1454-1464, 2013.

[19] A. Tantri, T. R. Vrabec, A. Cu-Unjieng, A. Frost, W. H. Annesley Jr., and L. A. Donoso, "X-linked retinoschisis: a clinical and molecular genetic review," Survey of Ophthalmology, vol. 49, no. 2, pp. 214-230, 2004.

[20] R. Brancato, U. Menchini, and A. Pece, "Idiopathic macular retinoschisis in the young subject associated with preretinal and prepapillary neovessels," Journal Français d'Ophtalmologie, vol. 7, no. 11, pp. 685-688, 1984. 


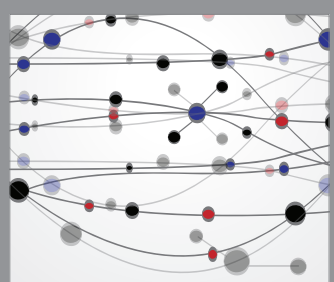

The Scientific World Journal
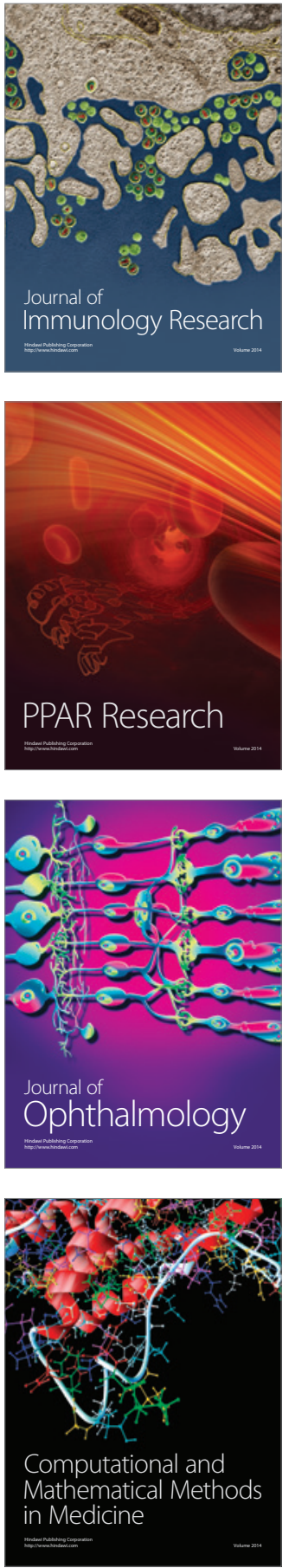

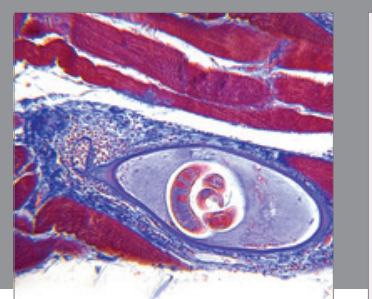

Gastroenterology Research and Practice
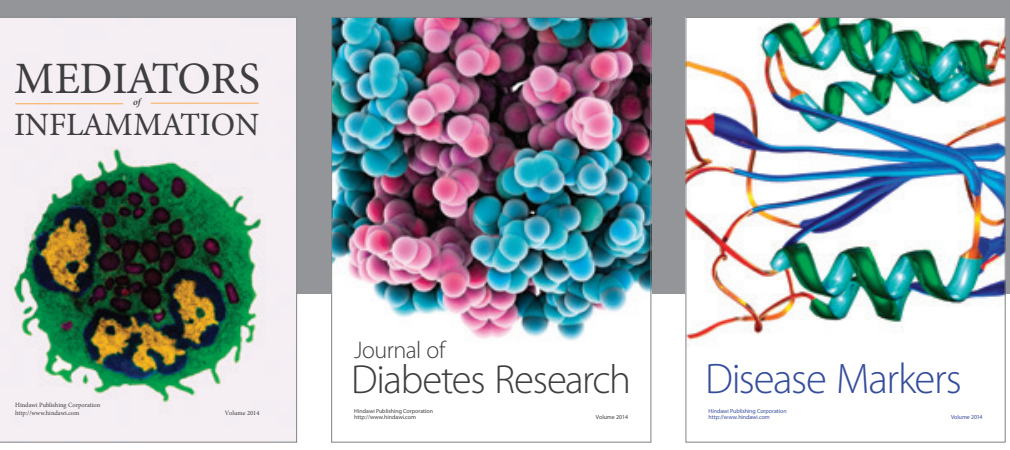

Disease Markers

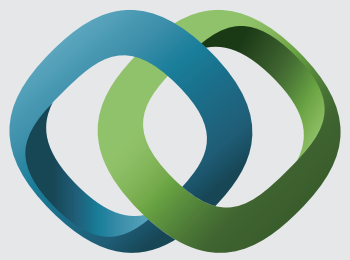

\section{Hindawi}

Submit your manuscripts at

https://www.hindawi.com
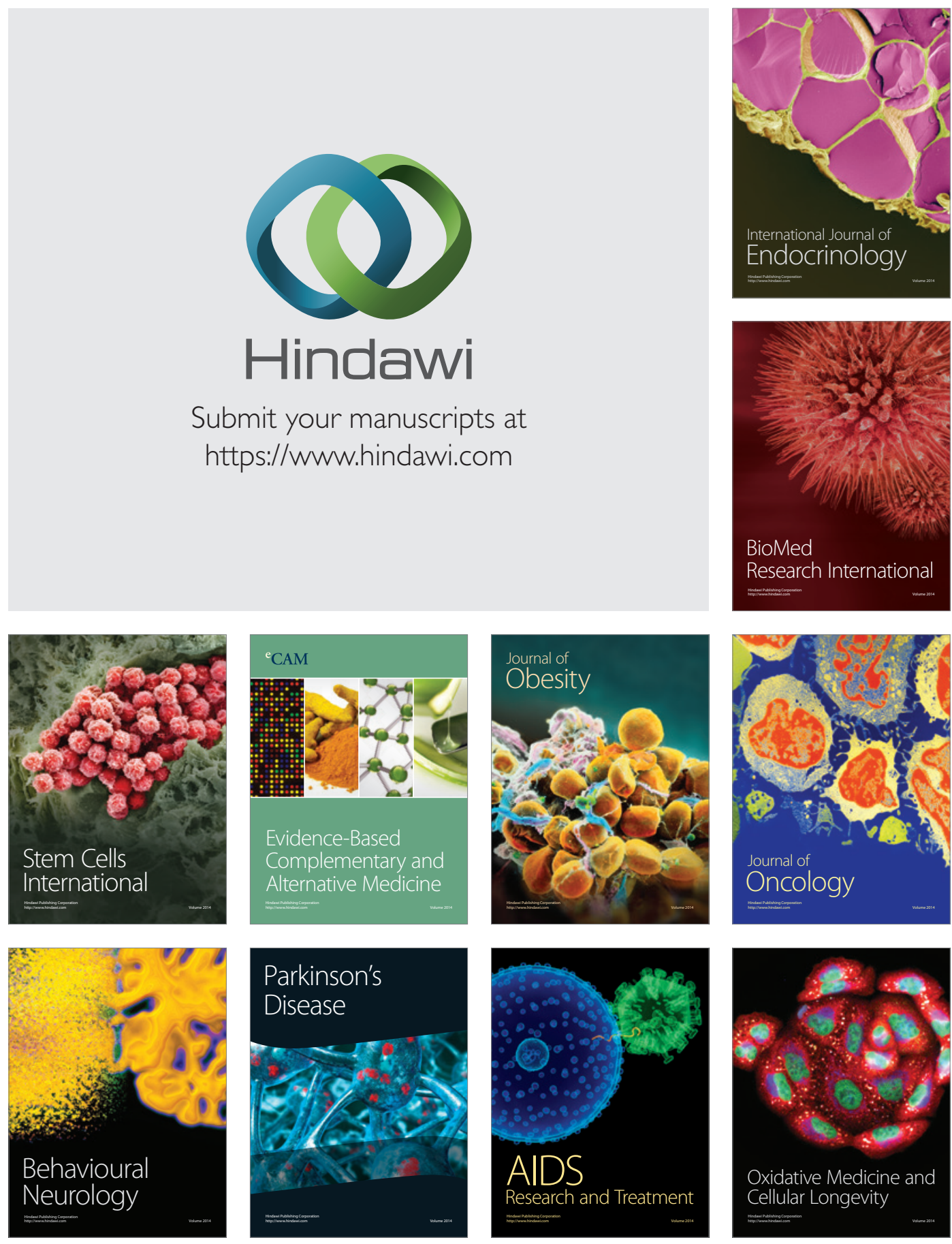